Old Dominion University

ODU Digital Commons

Bioelectrics Publications

Frank Reidy Research Center for Bioelectrics

2010

\title{
Frustrated Drift of an Anchored Scroll-Wave Filament and the Geodesic Principle
}

Marcel Wellner

Christian W.Zemlin

Old Dominion University, czemlin@odu.edu

Arkady M. Pertsov

Follow this and additional works at: https://digitalcommons.odu.edu/bioelectrics_pubs

Part of the Biochemistry, Biophysics, and Structural Biology Commons, and the Biomedical Engineering and Bioengineering Commons

\section{Repository Citation}

Wellner, Marcel; Zemlin, Christian W.; and Pertsov, Arkady M., "Frustrated Drift of an Anchored Scroll-Wave Filament and the Geodesic Principle" (2010). Bioelectrics Publications. 43.

https://digitalcommons.odu.edu/bioelectrics_pubs/43

\section{Original Publication Citation}

Wellner, M., Zemlin, C., \& Pertsov, A.M. (2010). Frustrated drift of an anchored scroll-wave filament and the geodesic principle. Phys Rev E Stat Nonlin Soft Matter Phys, 82(3 Pt 2), 036122. doi: 10.1103/PhysRevE.82.036122

This Article is brought to you for free and open access by the Frank Reidy Research Center for Bioelectrics at ODU Digital Commons. It has been accepted for inclusion in Bioelectrics Publications by an authorized administrator of ODU Digital Commons. For more information, please contact digitalcommons@odu.edu. 


\title{
Frustrated drift of an anchored scroll-wave filament and the geodesic principle
}

\author{
Marcel Wellner, ${ }^{1,2}$ Christian Zemlin, ${ }^{1}$ and Arkady M. Pertsov ${ }^{1}$ \\ ${ }^{1}$ Department of Pharmacology, SUNY Upstate Medical University, Syracuse, New York 13210, USA \\ ${ }^{2}$ Physics Department, Syracuse University, Syracuse, New York 13244-1130, USA \\ (Received 25 February 2009; revised manuscript received 3 September 2010; published 30 September 2010)
}

\begin{abstract}
We investigate anchored scroll-wave filaments in an excitable medium whose diffusivity matrix, including its determinant, is spatially nonuniform. The study is motivated by cardiological applications where scrollwave behavior in the presence of diffusivity gradients is believed to play an important role in the development of severe arrhythmias. A diffusivity gradient is expected to make the filament drift, unless drift is prevented ("frustrated") by anchoring to localized defects in the propagation medium. The resulting stationary filament is a geodesic curve, as demonstrated here in the case of a nonzero but constant gradient. That is, the diffusivity matrix has a determinant that varies in space, in contrast to what was assumed in earlier work. Here, we show that the filament shape results from a metric tensor of the form $(\operatorname{det} D) D^{-1}$, where $D$ is the diffusivity tensor. The filament's shape is solely determined by the diffusivity tensor and is independent of the equation's reaction terms. We derive the analytic solution for the filament and determine conditions for the existence of that solution. The theory is in excellent agreement with numerical simulations.
\end{abstract}

DOI: 10.1103/PhysRevE.82.036122

PACS number(s): 82.40.Ck

\section{INTRODUCTION}

A remarkable feature of three-dimensional (3D) excitable media is the existence of rotating scroll waves. In the heart such waves are pathological and usually lethal. Laboratory observations, as well as numerous computer simulations, are consistent with the presence inside the scroll of an unexcited tubular region, the filament. The scroll is organized and rotates around the filament, which is sometimes compared to the funnel of a tornado. For purposes of analysis, the filament is conveniently treated simply as a curve in space. Its shape and behavior are an excellent indicator for the configuration of the scroll as a whole $[1,2]$.

A scroll wave can be stable or unstable. For example, during episodes of ventricular fibrillation, scroll waves are constantly initiated and destroyed [3]. On the other hand, long-lived, steady-state scrolls have been observed [4], whose filaments are well represented (in simulations) by curves of fixed location and configuration. Such filaments were demonstrated, analytically and numerically, to be geodesic curves in 3D space, a property that was proved for steady-state filaments only. Steady state will be required in the present paper as well and will be enforced by anchoring the filament to small defects in the medium.

Static filaments are of particular interest in the context of the so-called "mother-rotor" hypothesis [5], according to which a stable scroll wave can set up and maintain ventricular fibrillation - a cause of sudden cardiac death. It is important for our work to note that if the filament of the mother rotor is anchored to small anatomical defects, its stability can be explained. If unanchored, a filament can drift within the heart tissue; here, we focus on diffusivity gradients as possible causes of drift.

Mathematically, scroll waves are of great interest as solutions of nonlinear diffusion-reaction equations. Rotating scrolls can be simulated on the computer with considerable realism by the use of such wave equations. Closed-form solutions are elusive, however, as they are for most nonlinear problems. Fortunately, there are nontrivial cases where the filament itself does have a closed-form solution, and this paper is devoted to one such example.

In earlier work we were able to characterize a static scroll-wave filament as a geodesic curve in three-space, with a simple formula for the relevant metric tensor. The present model, however, differs importantly from those found in our earlier studies. Here, an additional spatial gradient operator is incorporated in the diffusion term of the wave equation. Such a gradient is intended as a simplified representation of pathological variations that can exist in cardiac properties [6]; it has two special consequences. First, it causes the scroll to drift in the medium when the anchors are omitted [7]. Second, as explained below, even when the anchors are present the existence of an applied gradient invalidates our original formulation of the geodesic principle.

The reason for this lies in the diffusion term of the wave equation. The diffusivity of the medium can be represented by a $3 \times 3$ matrix $D$. A useful feature of $D$ is its determinant $\operatorname{det} D$; in all our previous work we had been dealing with a $\operatorname{det} D$ which was constant in space. That feature was compatible with a static (anchored) filament and with the existence of the above-mentioned geodesic principle, including the exact recipe for the metric tensor $g$, namely, $g=D^{-1}$ (the inverse of the diffusivity matrix). However, the gradient introduced here makes det $D$ variable in space, contrary to the earlier assumption. How should the recipe for $g$ be modified? At least in the model discussed here, the generalized metric is shown to be $g=(\operatorname{det} D) D^{-1}$ up to an arbitrary constant factor. Beyond the model of the present paper, the validity of our result is a tempting speculation that still needs to be proved in general. It is clear, nevertheless, that the formula automatically applies to the earlier (constant-determinant) cases as well.

Those latter cases are now well established, exactly in a class of models [8], and numerically in others. The geodesic principle has also been applied to a schematic representation of the cardiac ventricle [10]. A simple variational reformulation was proposed in Ref. [11]. A very general proof of the 
geodesic principle has subsequently been given $[12,13]$ for filaments that are not excessively curved. We note that Dierckx et al. [13] treated the equation of motion under nonconstant $\operatorname{det} D$ for a filament that is unanchored and thus allowed to drift. A meshing of these authors' context with ours would be of considerable interest.

There still remains a gap in generality. Can we have static filaments with nonconstant det $D$ ? Moreover, if we do, can a geodesic be derived for them? The answer is that a static filament is still easily obtained by anchoring to a small inhomogeneity. Furthermore, a geodesic can be derived even with a nonconstant $\operatorname{det} D$, and the geodesic includes the abovementioned prescription for $g$; our task in this paper is to demonstrate that fact.

Our model is briefly described as follows. Since an applied gradient can cause a scroll to drift and since, as already noted, our analysis must be confined to stationary filaments, we anchor both ends of the filament to surface defects on the opposite boundaries of a slab. We focus on one particularly simple yet nontrivial case selected because it is accessible to exact calculations; the model is described in Sec. II. Simulations of the model are essentially in perfect agreement with theory for all tested values of the gradient. There is much to be learned from such a simple setup:

(a) Our theoretical filament solution lies in a plane and so does the simulated filament.

(b) That plane is parallel to the gradient, contrary to what might be expected on the basis of the unanchored (drifting) situation, including scroll rings.

(c) A static simplified application of local filament dynamics on the one hand and the geodesic result on the other hand confirm one another in every detail.

(d) The set of solutions bifurcates with the applied gradient as the control parameter.

In short, our main result is to demonstrate that a geodesic principle is compatible with a spatially variable determinant for the diffusivity matrix. Perhaps equally important is an explicit recipe for the metric in a form that preserves earlier (constant-determinant) work.

A few technical remarks are in order at this stage. This paper adheres to some earlier restrictions on the existence of a geodesic. First, in the diffusion-reaction equation, the diffusion operator is time independent, although not in general space independent. Similarly, the reaction terms (membrane kinetics) have no explicit time or space dependence, although they do depend on space-time via the propagating variables. A third essential condition, as we have seen, is that the filament itself must be time independent; in particular, it should not drift or meander. Subject to those restrictions, the filament has another remarkable and perhaps unexpected feature: Its configuration is independent of the detailed reaction term. That is to say, knowledge of the diffusion operator and boundary conditions are in principle sufficient to determine the filament configuration - a result already found in Ref. [8].

In the treatment that follows, Sec. II sets up the frustrateddrift model; Sec. III introduces the geodesic approach; Sec. IV derives explicit solutions for the filament shape; Sec. V shows how the set of solutions bifurcates as the strength of the diffusivity gradient changes; Sec. VI examines the agree- ment between theory and simulation; Secs. VII and VIII propose a shortcut version of local filament dynamics and apply it to confirm the geodesic results, leading to a unique choice for the metric tensor normalization; lastly, Secs. IX and X discuss and summarize the results.

\section{MODEL}

With cardiac tissue in mind, we start from generalized FitzHugh-Nagumo equations for propagating variables $u, v_{1}, v_{2}, \ldots$, where $u$ diffuses and the $v$ 's do not,

$$
\begin{gathered}
\partial_{t} u-\partial_{i}\left(D_{i j} \partial_{j} u\right)+\Phi(u, \vec{v})=0, \\
\partial_{t} \vec{v}+\vec{\Psi}(u, \vec{v})=0
\end{gathered}
$$

( $t=$ time; $x_{1}, x_{2}, x_{3}=x, y, z=$ space). The reaction functions $\Phi$ and $\Psi$ do not depend on the $x_{i}$ or on $t$ except indirectly through the $u$ and $v$; the matrix $D_{i j}$ depends on space but not on time. It is assumed that systems (1) and (2) support scroll waves whose filament has positive tension, as explained in Ref. [14]; the details of $\Phi$ and $\Psi$ are otherwise of no concern. This paper deals with a stationary filament whose shape, as mentioned in Sec. I, is affected only by the space dependence of the $D_{i j}$ and by the boundary conditions.

The proposed model involves a spatially nonuniform diffusivity $D$. A simple realization of $D$ consists of a uniform isotropic matrix - the unit matrix - multiplied by a constant gradient of strength $G$ in the $x_{1}$ direction; $D$ is chosen to have unit normalization when $G=0$,

$$
D_{i j}=\left(1+G x_{1}\right) \delta_{i j} \quad(i, j=1,2,3 ; G \geq 0) .
$$

Equation (3) represents the model we shall study in detail.

For the diffusion operator in Eq. (1) we then have

$$
\partial_{i}\left(D_{i j} \partial_{j}\right)=(1+G x) \nabla^{2}+G \partial_{x} .
$$

The above is patterned after Ref. [7], where Eqs. (3) and (4) are postulated in a two-dimensional (2D) version dealing only with the $x_{1} x_{2}$ plane. The result is a deformed spiral with a drift whose direction depends on properties of the propagation medium. (For a simple application, see Ref. [15].) In two dimensions, the term $\left(1+G x_{1}\right) \nabla^{2}$ causes a deformation of the spiral in the $x_{1} x_{2}$ plane, but no drift, while the $G \partial_{1}$ term is responsible for the slanted drift but does not contribute to the deformation. Physically, we expect the scalar diffusivity $1+G x$ in Eq. (4) to be positive. Thus, we shall consider solutions only in the $x>-1 / G$ region.

In three dimensions, the above scenario is trivially replicated from Eq. (4) by a solution selected with propagating variables that are invariant in the $x_{3}$ direction, $\partial_{3} u=0, \partial_{3} \vec{v}$ $=0$. We then have a purely $z$ oriented scroll whose drifting filament keeps that same direction. (In this hypothetical case we are not concerned about whether the filament's rectilinear shape is stable or not.)

Keeping the 3D diffusivity as described above, we now "detrivialize" the $x_{3}$ behavior by anchoring the filament to two points on opposite boundaries of a slab-shaped medium. For simplicity both anchor points are in the $x_{1} x_{3}$ (or $x z$ ) plane, i.e., in a plane parallel to the diffusivity gradient. No 
generality is lost since for any given pair of anchor points such a plane exists. The shape and evolution of the filament are now no longer obvious. The anchoring procedure and its effects have been discussed in Refs. $[4,16]$, where, however, the drifting mechanism cannot be considered part of the diffusion term, as it is here.

\section{GEODESIC APPROACH}

The present section formulates the geodesic equation in terms of ordinary derivatives with respect to the element, $d l$, of the actual path. This formulation will be used later in order to solve for $x(l)$ and $z(l)$ along the geodesic curve, thus yielding the shape of the filament.

A metric $g_{i j}$ is needed to calculate a geodesic. Our previous research led us to

$$
g_{i j}=\left(D^{-1}\right)_{i j}
$$

up to a constant factor; $D^{-1}$ is the inverse of the diffusivity matrix. However, as mentioned above, we now relax the constancy assumption for det $D$, thus allowing

$$
\partial_{i} \operatorname{det} D \neq 0 \text {. }
$$

That generalization is made possible by the postulate, namely,

$$
g_{i j}=(\operatorname{det} D)\left(D^{-1}\right)_{i j}
$$

to replace Eq. (5). The motivations for Eq. (7), besides its simplicity, are its reduction to Eq. (5) under the previous assumption of a constant det $D$, its seemingly exact verification in simulations, and, most conclusively, the analytic and numerical results in Sec. VIII, Appendix D, and Fig. 9 further on. Partial justification is also provided in Appendix A.

From here on we work within the simple model of Eq. (3), where

$$
\operatorname{det} D=\left(1+G x_{1}\right)^{3}
$$

and where $\left(D^{-1}\right)_{i j}=\left(1+G x_{1}\right)^{-1} \delta_{i j}$. Postulate (7) reads, in short,

$$
g_{i j}=f\left(x_{1}\right) \delta_{i j},
$$

where

$$
f(x)=(1+G x)^{2} .
$$

We are now ready to examine the consequences of hypothesizing a geodesic principle for the filament. Parametrically, in terms of a path coordinate $\sigma$ with element $d \sigma$ $=\sqrt{g_{i j} d x_{i} d x_{j}}$ along the curve, we have [8]

$$
g_{k i} \ddot{x}_{i}=\left(\frac{1}{2} \partial_{k} g_{j m}-\partial_{m} g_{j k}\right) \dot{x}_{j} \dot{x}_{m},
$$

where a dot stands for $d / d \sigma, d \sigma=\sqrt{g_{i j} d x_{i} d x_{j}}$.

The first main consequence of postulating the geodesic is that we find filaments with both anchors in the $x z$ plane, which lie entirely in that plane. Indeed, Eq. (11) has solutions, i.e., filaments, with $y=0$ everywhere and with both anchors in the $x z$ plane. That result is somewhat unexpected on the basis of unanchored filaments, whose observed drift is not coplanar with the applied gradient [7]. Our numerical analysis (Sec. VI) confirms the coplanarity. We have encountered no filaments that deviate from the plane, although we have not been able to rule out such solutions.

In order to find the full coplanar solutions of Eq. (11), we note that $\partial_{i} \rightarrow 0$ unless $i=1$, that $g_{i j}$ is diagonal, and that the solutions are restricted to the $y=0$ plane as explained. Thus, we have from Eqs. (10) and (11)

$$
\begin{gathered}
\ddot{x}=\frac{d f / d x}{2 f}\left(-\dot{x}^{2}+\dot{z}^{2}\right), \\
\ddot{z}=-\frac{d f / d x}{f} \dot{x} \dot{z}
\end{gathered}
$$

for $k=1$ and 3 , respectively. In terms of the element of ordinary length $d l=\sqrt{d x^{2}+d z^{2}}$, we have

$$
\begin{gathered}
\frac{d}{d \sigma}=\frac{1}{\sqrt{f}} \frac{d}{d l}, \\
\frac{d^{2}}{d \sigma^{2}}=\frac{1}{f} \frac{d^{2}}{d l^{2}}-\frac{d f / d x}{2 f^{2}} \frac{d x}{d l} \frac{d}{d l} .
\end{gathered}
$$

The result from Eqs. (12) and (13) is

$$
\begin{aligned}
& x^{\prime \prime}=\frac{d f / d x}{2 f}\left(z^{\prime}\right)^{2}, \\
& z^{\prime \prime}=-\frac{d f / d x}{2 f} x^{\prime} z^{\prime},
\end{aligned}
$$

where the prime indicates $d / d l$. A parametrization constraint,

$$
\left(x^{\prime}\right)^{2}+\left(z^{\prime}\right)^{2}=1,
$$

follows from the definition of $d l$. The form of Eqs. (16) and (17) confirms the essential role played by the function $f$, that is to say, by $\operatorname{det} D$.

In summary, Eqs. (16)-(18) are an expression of the geodesic postulate. The form of $f$ has been left open in order that we may later find the consequence of departing somewhat from the exact Eq. (10). The solution of Eqs. (16)-(18) is discussed next.

\section{FILAMENT SOLUTION}

With $f(x)$ as in Eq. (10), we obtain directly from Eq. (17)

$$
z^{\prime}=\frac{A}{x+1 / G}
$$

where $A$ is the integration constant. Inserting this formula for $z^{\prime}$ into Eq. (18) gives

$$
\left(x^{\prime}\right)^{2}+\frac{A^{2}}{(x+1 / G)^{2}}=1,
$$

whose solution is readily verified to be

$$
x+1 / G=\sqrt{l^{2}+A^{2}} .
$$

Another integration constant has been avoided here through fixing the origin of $l$ at $x^{\prime}=0$. Positivity of the diffusion 


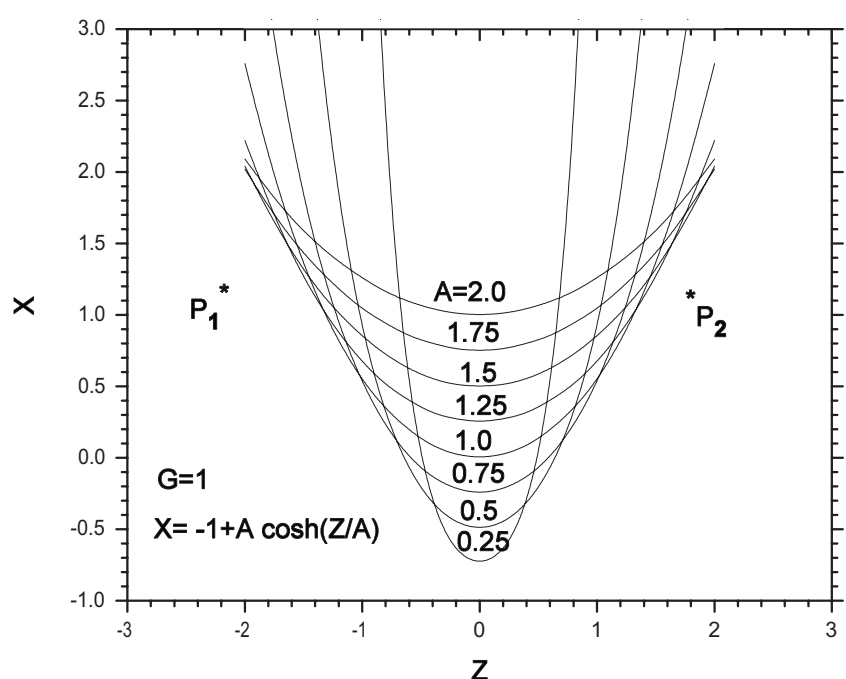

FIG. 1. Representative filament solutions. This is a plot of Eq. (23) with $G=1, z_{0}=0$, and several values of $A$. There are no solutions for $x<1$. Note the wedge-shaped envelope of the filaments. Its angle is determined by Eqs. (24) and (25); no solutions anchored simultaneously to points located like $P_{1}$ and $P_{2}$ have been found. Note also the twofold nature of solutions with two given anchor points.

matrix requires the positive square root in Eq. (21). From Eq. (19) we then get

$$
z=A \sinh ^{-1} \frac{l}{A}+z_{0}
$$

An adjustment of $z_{0}$ is equivalent to a shift in the origin of $z$, and therefore we take $z_{0}=0$ without any essential loss in generality. Eliminating $l$ yields

$$
x=-\frac{1}{G}+A \cosh \frac{z}{A} .
$$

This is the equation for the filament according to the geodesic principle. Figure 1 shows a representative set of filaments, with $z_{0}=0$ and $G=1$. No filament exists where $x<$ $-1 / G$, i.e., where the diffusivity in Eq. (3) is negative.

We note the envelope of solutions, a wedge composed of two straight lines,

$$
z= \pm \frac{\lambda}{\cosh \lambda}\left(x+\frac{1}{G}\right),
$$

where $\lambda \approx 1.20$ is the solution of

$$
\tanh \lambda=\frac{1}{\lambda}
$$

(see also Sec. V).

Two filaments with different $A$ values intersect at two points, and thus a given pair of anchor points, remarkably, gives a pair of filament solutions rather than a unique one. Of the two solutions, the one with the shorter total geodesic path is found to be stable. Little is known at present about the properties of the other solution.

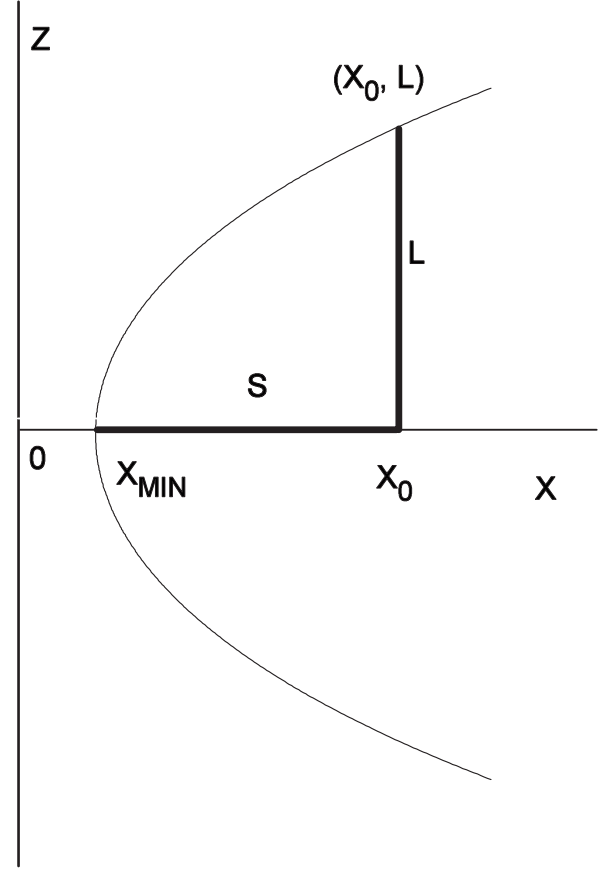

FIG. 2. Notation used in calculating the midpoint deflection of the filament. One anchor point is shown at $(x, z)=\left(x_{0}, L\right)$. The filament's midpoint is at $x=x_{\min }$ and its deflection is $s=x_{0}-x_{\min }$, defined as a positive distance.

For some pairs of would-be anchor points, say $P_{1}$ and $P_{2}$ in Fig. 1, we find no filament solutions, even by shifting the $z$ coordinate. Together, all shifts of $z$ give rise to a family of envelope wedges; $P_{1}$ and $P_{2}$ must be inside the same wedge in order to produce an anchored filament. Simulations must ensure in addition that enough turns of the scroll (at least one or two) are included around the filament within the computational boundaries.

\section{FILAMENT DEFLECTION AND ITS BIFURCATION}

In preparation for Sec. VI, which compares simulation and theory, we derive some additional properties of the solution, mainly (a) dependence of the maximum filament deflection on the gradient $G$ and (b) some bifurcation facts concerning that deflection. For convenience let us consider the symmetric geometry of Fig. 2, where the anchor points have coordinates $(x, y, z)=\left(x_{0}, 0, \pm L\right)$ for some chosen slab thickness $2 L$. Under a diffusivity gradient in the $x$ direction, the theory is seen to be consistent with a stable filament in the $x z$ plane.

The filament's midpoint deflection from rectilinear is

$$
s=x_{0}-x_{\min },
$$

where $x_{\min }$ is the minimum $x$ coordinate on the filament (see Fig. 2). The quantity $s$ is a practical measure of how strongly $G$ affects the filament and numerically useful for comparing theory and simulation. We assume a positive gradient $G>0$ without loss of generality since the system is invariant under $G \rightarrow-G, x_{0} \rightarrow-x_{0}$, and $s \rightarrow-s$. The filament is then deflected in the $-x$ direction. 
A brief digression will now produce, from theory, $s$ as a function of $L, G$, and $x_{0}$. Equation (23), expressed at the anchor point, reads

$$
x_{0}=-\frac{1}{G}+A \cosh \frac{L}{A} .
$$

On the other hand, at $z=0$, Eq. (23) reads

$$
x_{\min }=-\frac{1}{G}+A
$$

or, with Eq. (26),

$$
\frac{s}{L}=\frac{A}{L}\left(\cosh \frac{L}{A}-1\right),
$$

written in dimensionless form; $A$ depends on $x_{0}$.

In the special case $x_{0}=0$, the solution of Eq. (27) already exhibits in a simple graphical way the existence of a bifurcation, as seen later in Fig. 5 (in that figure, $G^{\prime}$ then reads $G)$. Tangency of the two curves gives the bifurcation point.

We now return to the deflection $s$ for general values of $x_{0}$. The quantity $A / L$ still must be eliminated from Eqs. (27) and (29). No explicit formula is available, but the desired dependence $s=s\left(L, G, x_{0}\right)$ is readily obtained in terms of an auxiliary gradient,

$$
G^{\prime}=\frac{G}{1+x_{0} G} .
$$

Equation (27) now reads

$$
\frac{1}{G^{\prime} L}=\frac{A}{L} \cosh \frac{L}{A},
$$

allowing us to plot $s / L$ against $G^{\prime} L$ parametrically from Eqs. (29) and (31) with $L / A$ as the running parameter. The result is a two-valued curve for $s / L$. Both branches are shown in Fig. 3; they meet at the bifurcation point B,

$$
\left(G_{0}^{\prime} L, s_{0} / L\right) \approx(0.662743,0.675323) .
$$

These are universal numbers in the sense that they do not depend on any parameter of the model if the unperturbed diffusivity has unit normalization. The lower branch, reachable by stable simulations, is displayed in more detail in Fig. 4 ; accessibility of the higher branch is problematical. Likewise, no stable scroll wave has been produced with $G^{\prime} L$ above its bifurcation value $G_{0}^{\prime} L$.

How the bifurcation occurs is most directly visualized when solving Eq. (31) for $L / A$ in terms of $G^{\prime} L$. Plotting $(L / A)\left(1 / G^{\prime} L\right)$ and $\cosh (L / A)$ as functions of $L / A$ but with fixed $G^{\prime} L$, we look for an intersection of those two curves. A case with two intersections $P, Q$ is shown in Fig. 5. Point $P$ pertains to the lower branch of $s$ in Fig. 3 and point $Q$ to the higher one. With a decreasing slope, $1 / G^{\prime} L$, of the straight line, the two solutions merge and then disappear. At bifurcation their slopes are equal,

$$
1 /\left(G^{\prime} L\right)=\sinh (L / A) .
$$

Solving Eqs. (31) and (33) for the bifurcation values $A_{0}$ and $G_{0}^{\prime}$ of $A$ and $G^{\prime}$, we find



FIG. 3. Dimensionless midpoint deflection $s / L$ of the filament as a function of the dimensionless gradient $G^{\prime} L$. At $G^{\prime} L=0$, the lower branch has slope $1 / 2$. The upper branch behaves as $\left(G^{\prime} L\right)^{-1}$ when $s \rightarrow 0$. The two branches meet at the bifurcation point $\mathrm{B}$. Figure 4 displays the lower branch in more detail. Solutions appear to be stable or unstable according to whether they are on the lower or upper branch, respectively.

$$
\tanh \frac{L}{A_{0}}=\frac{A_{0}}{L}
$$

whose solution is $L / A_{0}=\lambda \approx 1.199678$ [ $\lambda$ from Eq. (25)], with $G_{0}^{\prime} L$ given in Eq. (32).

In what way does the actually realized minimum (intersection $P$, smaller $L / A$ in Fig. 5) differ from the unrealized minimum (intersection $Q$, larger $L / A$ )? The answer, illustrated in Fig. 6, is that, for any given $G L$, the smaller $L / A$ is associated with the shorter geodesic path length $\mathcal{L}=\int d \sigma$ be-

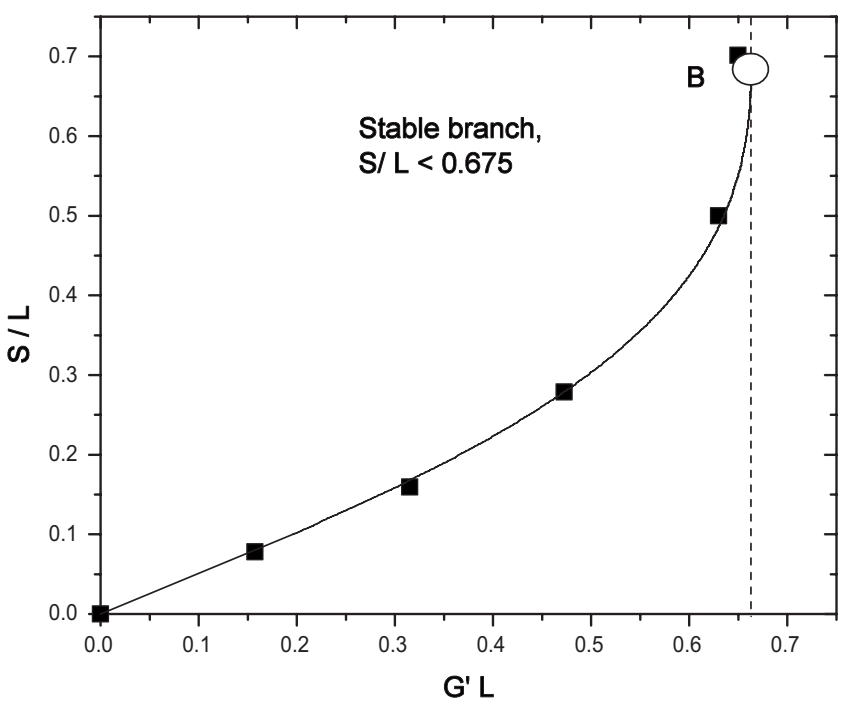

FIG. 4. Lower branch of Fig. 3. Theory, solid curve; simulation, black squares; and theoretical bifurcation point, hollow circle at B, with coordinates given in Eq. (32). Filament solutions are found only to the left of the vertical dashed line. 


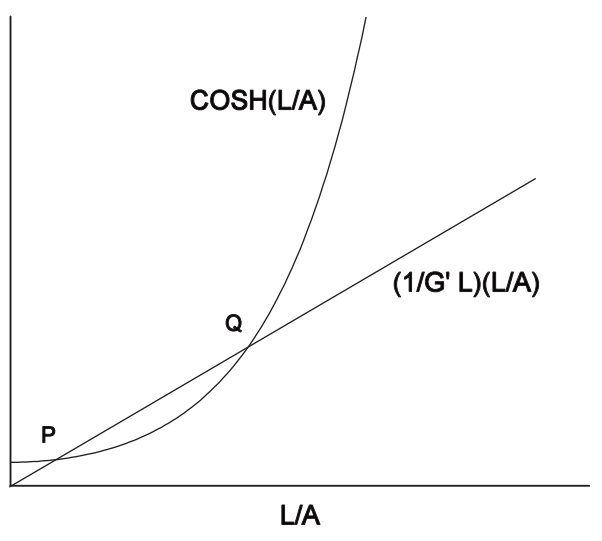

FIG. 5. Bifurcation arises when $G^{\prime}$ becomes large enough to make the straight-line tangent to the cosh curve. Relative to the point of tangency, the upper segment of that curve corresponds to the lower branch of $s$ in Fig. 3 and vice versa.

tween the anchors. Both filaments obey the same differential geodesic equation and both are therefore minimal under short-wave deformations (local minima). However, in Fig. 6 the lower branch corresponds to the global minimum: it is minimal under long-wave deformations as well. The two branches have been verified to involve the larger or smaller $L / A$, respectively. How to plot the value of $\mathcal{L}$ is briefly indicated in Appendix C.

\section{SIMULATIONS VERSUS THEORY}

This section presents in Fig. 7 the result of our simulations for the filament shape, $x$, as a function of $z$ [Eq. (23)]



FIG. 6. Total dimensionless geodesic path length as a function of dimensionless gradient (cf. Appendix C). For the sake of illustration we take the anchors at $x$ coordinate $x_{0}=0$. The main interest of this curve is to show that, out of two filament solutions with the same $G$, the physically realized filament chooses the shorter of the two path lengths. This statement was verified from the value of $A$ used in the parametric plotting: the smaller of the two possible $L / A$ values (cf. Fig. 5) yields a point on the lower branch. That branch starts at $(0,2)$ and slowly falls to $(0.663,1.81)$ at the cusp.
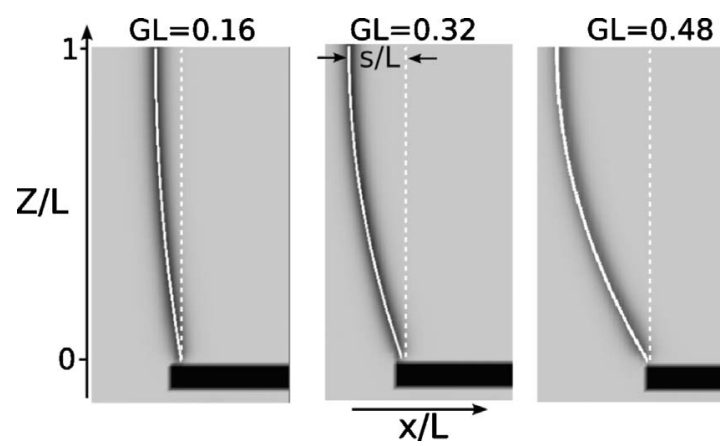

FIG. 7. The $0<z<L$ half of the filament curve, displayed in the $y=0$ plane. The anchor is the dark inexcitable rectangle at the bottom of the figure. The simulation result is shown as a diffuse dark curve; theory gives the white curve. The match between the two curves is quite striking. The white dashed vertical line is the unperturbed filament. The horizontal and vertical scales in this figure are the same. Details are given in the text of Sec. VI.

for three representative values of $G$. Only the bottom half of the filament is shown since the top half is just its symmetric counterpart. The white curve is plotted from Eq. (23), whereas the more diffuse dark curve is the simulated filament. We see an excellent agreement between theory and computation, even for $G L=0.48$, which is already fairly close to bifurcation, $G L \rightarrow G_{0} L \approx 0.66$, the strongest gradient that still supports a stable filament.

Some computational details are as follows. The simulations converge in a feasible amount of time with $x_{0} G \ll 1$. Therefore, looking back at Eq. (48), we take $x_{0}=0$, that is to say, $G^{\prime}=G$ in our computational examples. We used Barkley's [17] particular realization of Eqs. (1) and (2),

$$
\begin{gathered}
\epsilon \partial_{t} u=u(1-u)[u-(v+b) / a]+\partial_{i}\left(D_{i j}\right) u, \\
\partial_{t} v=u-v,
\end{gathered}
$$

with parameter values $a=0.9, b=0.05$, and $\epsilon=0.02$ chosen for intermediate excitability. Computations used Euler's method, a 32-core Beowulf cluster, and a $120 \times 60 \times 120 x y z$ grid. Boundary conditions were zero flux; space and time steps were $\Delta x=0.25$ and $\Delta t=0.006$. The filament is defined by those voxels where $u$ changes by less than (typically) 0.65 over a full scroll rotation. By comparison, $u$ never exceeds about 1.00 anywhere in our simulations. Anchoring is to the tip of a narrow cylinder (diameter $\approx 8$ pixels) protruding into the medium and without diffusive coupling to it. Such a setup minimizes the scroll's interaction with the boundary. The half-distance $L$ between anchoring points is taken to be $L=15.75$.

Returning to Fig. 4, the computational maximum deflection $s$ as a function of the gradient $G$ has been displayed as data points in that figure. There is, here also, striking agreement with theory. The computational specifications are the same as above.

\section{LOCAL STATICS IN A NONUNIFORM MEDIUM}

For another analytic approach to filament calculation, we use a heuristic method that simplifies local dynamics [2], 


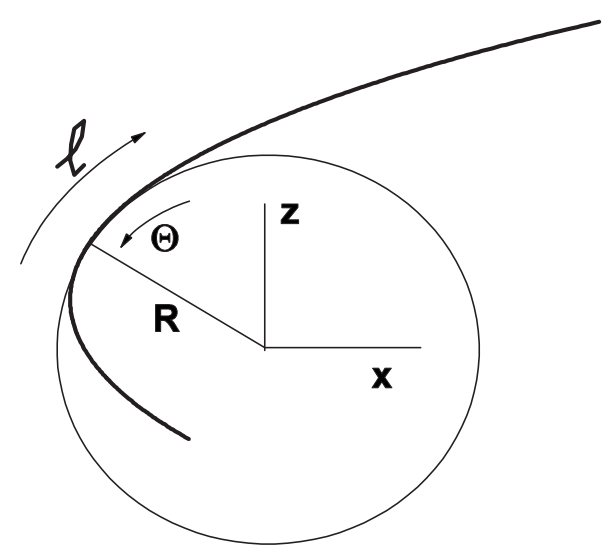

FIG. 8. Notation scheme for $l, \Theta, R, x$, and $z$ on an osculating circle. The heavy curve is the filament, which lies in the plane of the figure.

here specialized to a static filament in a two-dimensional plane. The main purpose is to provide support other than computational for the choice of $f(x)$ in Eq. (10). Section IX discusses in what way the present method constitutes a shortcut to the theoretical solution. We just require that, at any point on the filament, the diffusion operator should not include first-order differentiation perpendicular to the filament. This simple rule presupposes a diffusion matrix which is locally isotropic, i.e., proportional to the unit matrix, as in Eq. (9) (see Sec. IX).

For implementation, we use polar coordinates $(r, \theta)$ in the plane of the filament, with $x=r \cos \theta, z=r \sin \theta$. We represent the filament locally by its osculating circle, whose center is taken for now to be the origin of coordinates. The circle has radius $R$ and is tangent to the filament at some point, $(r, \theta)=(R, \Theta)$; the radius $R$ equals the filament's curvature at that point. The notation is illustrated in Fig. 8.

In the diffusion operator, we isolate the term in $\partial / \partial r$, i.e., the term involving the first derivative normal to the filament. At the filament $(r=R)$ the overall perpendicular differentiation term must be absent. Absence of $\partial / \partial r$ is a rule motivated as follows: In a small vicinity of a filament point and in the coordinates of Fig. 8, the diffusion operator should have the form that is known to produce a nondrifting scroll. This means zero gradient except possibly in the direction of the filament. In short, local dynamics requires that $\partial / \partial r$ have zero coefficient: both contributions to the convection, one due to filament curvature and the other due to diffusion, should mutually cancel. We are dealing here with an intuitive working assumption that will be treated as exact in what follows.

We rewrite the diffusion operator in polar coordinates as

$$
\partial_{i}\left(D_{i j} \partial_{j}\right)=D_{i j} \partial_{i} \partial_{j}+\left(\partial_{i} D_{i j}\right) \partial_{j},
$$

where

$$
D_{i j} \partial_{i} \partial_{j}=\mathcal{D}_{r r} \partial_{r}^{2}+\mathcal{D}_{r \theta} \partial_{r} \partial_{\theta}+\mathcal{D}_{\theta \theta} \partial_{\theta}^{2}+\mathcal{D}_{r} \partial_{r}+\mathcal{D}_{\theta} \partial_{\theta}+T
$$

$$
\left(\partial_{i} D_{i j}\right) \partial_{j}=\mathcal{E}_{r} \partial_{r}+\mathcal{E}_{\theta} \partial_{\theta}+U
$$

Here, $T$ and $U$ involve $\partial_{2}^{2}$ but no first derivative $\partial_{2}$; therefore, the $x_{2}(=y)$ dimension is of no interest in most of what follows.

The $\mathcal{D}$ 's and $\mathcal{E}$ 's are polynomials in $\cos \theta$ and $\sin \theta$. Along a fixed circle, here $r=R$, we have $\theta=-l / r$ in the geometry of Fig. 8, and therefore $\cos \theta=-z^{\prime}$ and $\sin \theta=x^{\prime}$, where the prime still indicates $d / d l$.

Returning to Eqs. (38) and (39), we only need expressions for $\mathcal{D}_{r}$ and $\mathcal{E}_{r}$ at the osculating point; for completeness all seven $\mathcal{D}$ 's and $\mathcal{E}$ 's are listed in Appendix B. We have

$$
\mathcal{D}_{r}=\frac{1}{R}\left[D_{11}\left(x^{\prime}\right)^{2}+2 D_{13} x^{\prime} z^{\prime}+D_{33}\left(z^{\prime}\right)^{2}\right],
$$

where $1 / R$ is the local positive curvature of the filament. Equation (40) gives the curvature-induced convection. Similarly, Eq. (39) involves

$$
\mathcal{E}_{r}=-\left(\partial_{i} D_{i 1}\right) z^{\prime}+\left(\partial_{i} D_{i 3}\right) x^{\prime} .
$$

This is the diffusivity-induced convection. The relative signs of Eqs. (40) and (41) are as they occur in the coefficient of $\partial / \partial r$. Zero convection means

$$
\mathcal{D}_{r}+\mathcal{E}_{r}=0
$$

at the filament. This equation, amounting to local statics, will next be enforced at all points of the filament. Based on confirmation from the geodesic equation as well as from the numerical results of Sec. VI further on, we shall treat Eq. (42) as exact. We make no claims of validity, however, for any $D_{i j}$ of more general form than Eq. (3). Section IX has an additional comment on that limitation. In summary, Eq. (42), with details from Eqs. (40) and (41), is the main result of this section.

\section{LOCAL STATICS OF FRUSTRATED DRIFT}

This section applies Eq. (42) to derive the filament shape. We start with Eq. (40), in which we use the standard formula for the curvature,

$$
\frac{1}{R}=z^{\prime} x^{\prime \prime}-x^{\prime} z^{\prime \prime}
$$

Also in Eq. (40), we have

$$
D_{11}=D_{33}=1+G x, \quad D_{13}=0 .
$$

Thus, we have

$$
D_{r}=(1+G x)\left(z^{\prime} x^{\prime \prime}-x^{\prime} z^{\prime \prime}\right)\left(x^{\prime 2}+z^{\prime 2}\right)
$$

or, since the last factor is unity,

$$
D_{r}=(1+G x)\left(z^{\prime} x^{\prime \prime}-x^{\prime} z^{\prime \prime}\right) .
$$

In Eq. (41) we have

$$
\partial_{i} D_{i 1}=\partial_{x}(1+G x)=G,
$$

$$
\partial_{i} D_{i 3}=0,
$$




$$
\mathcal{E}_{r}=-G z^{\prime} .
$$

In conclusion, Eq. (42) reads

$$
(1+G x)\left(z^{\prime} x^{\prime \prime}-x^{\prime} z^{\prime \prime}\right)-G z^{\prime}=0,
$$

with the constraint

$$
x^{\prime 2}+z^{\prime 2}=1 \text {. }
$$

But this last pair of equations is precisely equivalent to the geodesic result, as can be seen by combining Eqs. (16)-(18). The equivalence is contingent on $f(x)=(1+G x)^{2}$ [Eq. (10)]. In short, if the geodesic principle is exact, so is local filament statics; their results coincide for the appropriate normalization of $g_{i j}$.

\section{DISCUSSION}

The original analytic derivation of the geodesic principle for scroll-wave filaments, namely, in deformed anisotropy $[8,9]$, made use of a transformed coordinate system $X_{i}$ $=X_{i}\left(x_{1}, x_{2}, x_{3}\right)(i=1,2,3)$ in which the filament is manifestly linear provided only that it is stable and unique under the boundary conditions employed; the inverse transformation then yields the actual filament. What is the outlook for that type of proof in the present context? Linearity of the transformed filament would arise from one of at least two possibilities:

(a) There is a flat $X$ space, with metric $\delta_{i j}$; it implies the existence of cylindrical scrolls, each with its rectilinear filament. In order for a flat $X$ system to be possible, the original system must already have zero intrinsic (RiemannChristoffel) curvature. However, such is not the case in frustrated drift. Figure 1 shows pairs of filaments involving two intersections - a property of geodesics in curved spaces only. A single Riemann-Christoffel tensor element [18], $R_{1212} \neq 0$, already confirms the nonzero curvature. Therefore, we fall back on the assumption that

(b) the $X$ space is sufficiently symmetric with respect to a straight line, e.g., a coordinate axis; case (a) is a special instance of this. Symmetry was used in the original proof of the geodesic principle under deformed anisotropy. There, the metric has nonzero intrinsic curvature, and in particular it can be verified that, in the coordinate system of that article, $R_{3131} \neq 0$. A symmetrizing transformation may well exist in the present case as well.

Is the present treatment mathematically exact? The answer must recognize that a scroll-wave filament cannot, in general, be defined precisely-it is a "diffuse" concept. In contrast, the filament as conceived in the present research is a curve, i.e., it has perfect sharpness. In addition, it is time independent. In practice, a time-independent filament is a tube rather than a line. For example, as analyzed in Ref. [8], the tube's thickness changes along its length and, more importantly, depends on its definition in terms of the propagating variables (see also Sec. VII above). We conclude that, in general, the theoretical filament is a representation rather than an exact description of the actual filament. Here, that representation has been calculated exactly.

As concerns this paper's shortcut to local filament statics, we note that even in the nonmeandering periodic case, there exist many ways to define a time-independent filament. This is in contrast to the $2 \mathrm{D}$ case, where a nonmeandering periodic spiral has a clear-cut rotation center. Therefore, it seems reasonable to make the following conjecture: Since the validity of a filament curve can be determined only within the approximate filament tube, our local filament dynamics provides as good a representation as any that one might, in principle, expect.

It should be noted, incidentally, that in Refs. [8,9] the filament's curvature has not been an issue: theory accommodates up to infinite curvatures (sharp corners), and simulations do so as well so far, to voxel precision.

Finally, we caution that local statics as described in Sec. IV breaks down in a locally anisotropic medium-not a concern in the present model. Indeed, the osculating-circle construction of Fig. 8 would need to be amended in that general case. Such a consideration in no way precludes the general validity of the geodesic principle in the form of Eq. (11).

\section{SUMMARY}

We have examined a scroll wave that propagates in a parallel slab whose diffusivity matrix has a constant gradient parallel to the boundaries. An added feature to this otherwise well-known model is filament anchoring to the slab's boundaries. No twist of the scroll has been considered since it would be expected to decay with time in the present setup. Our purpose has been to extend the geodesic principle for the filament to the nonconstant determinant case. We wanted to know whether a steady-state filament exists, and if so, whether it lies in a plane and whether that plane is in the direction of the unanchored drift, the direction of the gradient, or any other alternative. Our work demonstrates that

(a) There exists a steady-state filament whose shape is calculable exactly;

(b) That filament lies in the plane of the diffusivity gradient;

(c) It obeys a geodesic principle whose metric has the (nonconstant) determinant of the diffusivity as a factor;

(d) In the present case at least, geodesic and local dynamics (local statics) agree in their results; and

(e) Our numerical study shows excellent agreement with theory.

In conclusion, three independent approaches, namely, the geodesic principle, numerical simulation, and local statics, converge to the same filament configuration and frustrateddrift behavior.

In addition, we unexpectedly found the following:

(a) The deflection of the filament from a straight line displays a bifurcation in the strength of the diffusivity gradient; one branch, which is not studied in detail here, appears to be unphysical.

(b) Fundamental upper bounds exist for the filament deflections and for the gradient.

(c) The bifurcation implies the existence of two geodesic paths between the anchor points and applies to the total geodesic path length. The shorter path is associated with the stable solution. 


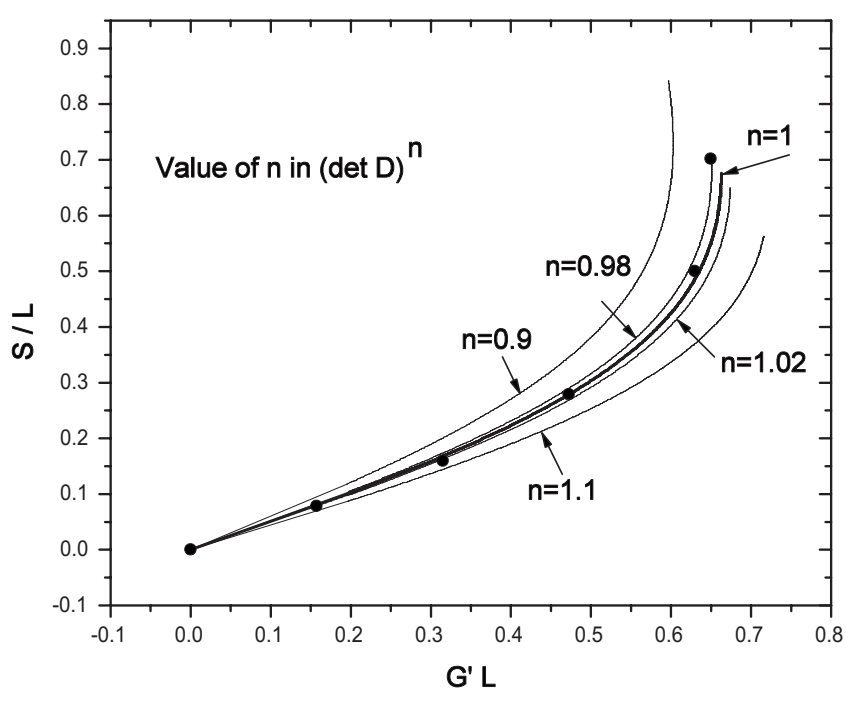

FIG. 9. A reexamination of Fig. 4, showing filament midpoint deflection versus applied gradient. The points are from computer simulations of the scroll wave. The curves are from a family of geodesic theories, in which the metric normalization is $(\operatorname{det} D)^{n}$ for various values of $n$. The present study postulates $n=1$ (heavy curve); the figure confirms that $n$ is between 0.98 and 1.02 .

\section{ACKNOWLEDGMENTS}

We are indebted to Olivier Bernus, Hans Dierckx, and Henri Verschelde for their valuable input. We thank the anonymous referees for their useful suggestions. Our work was supported by National Institutes of Health Grants No. 2RO1HL07176205A2 and No. 5RO1HL7163505 (for A.M.P. and M.W.) and by American Heart Association Grant No. AHA0830018N (for C.Z.).

\section{APPENDIX A: NORM OF THE METRIC TENSOR FROM SCALING}

The basic postulate [Eq. (7)] is a special case of

$$
g=(\operatorname{det} D)^{n} D^{-1}
$$

a form that will now be derived analytically. The special value $n=1$ is obtained in Sec. VIII, Appendix D, and Fig. 9.

We start from the previous result, where det $D=$ const and where we had $g=($ const $) D^{-1}$ or equivalently

$$
g=[F(\operatorname{det} D)] D^{-1},
$$

with $F$ being an arbitrary nonzero function. We take (without loss of generality so far) the form of $F$ to be model independent. In this paper we ask what is the result of keeping Eq. (A2) as written even if $\operatorname{det} D$ is no longer constant. This assumption has the virtue of enforcing consistency with previous work. Within that choice, it is now possible to show that $F$ is restricted to some power of $\operatorname{det} D[\mathrm{Eq}$. (A1)]. A strategy that leads to Eq. (A1) is as follows:

(a) As $D$ is varied, let Eq. (A2) remain valid;

(b) Require the diffusion-reaction equation [Eq. (1)] to keep its form; and

(c) Require the geodesic equation to keep its form.
Here, we shall not consider a general transformation that varies $D$. Rather, we limit ourselves to only one family of modified $D$ 's, obtained through scale transformations. Consider such a transformation, involving an arbitrary parameter $\lambda$,

$$
\begin{gathered}
(x, y, z) \rightarrow(\lambda)(x, y, z), \\
\partial / \partial(x, y, z) \rightarrow(1 / \lambda) \partial / \partial(x, y, z) .
\end{gathered}
$$

In order to preserve the diffusion-reaction equation [Eq. (1)] we must scale $D$ and therefore its determinant according to

$$
D \rightarrow \lambda^{2} D, \quad \operatorname{det} D \rightarrow \lambda^{6} \operatorname{det} D .
$$

Therefore, Eq. (A2) scales to

$$
g \rightarrow\left[F\left(\lambda^{6} \operatorname{det} D\right)\right]\left(\lambda^{2} D\right)^{-1} .
$$

Next, we require that the geodesic equation [Eq. (11)] be preserved as well. In that equation we see that $g$ can be scaled by a constant, say $g \rightarrow \mu g$. Then, Eq. (A2) reads

$$
\mu g=\left[F\left(\lambda^{6} \operatorname{det} D\right)\right]\left(\lambda^{2} D\right)^{-1} .
$$

Inserting Eq. (A2) in the above and multiplying by $D$, also denoting $\lambda^{2} \mu$ by $A, \lambda^{6}$ by $B$, and the variable det $D$ by $X$, we have

$$
A F(X)=F(B X) .
$$

This is a functional equation for $F$, to be solved as follows: Eq. (A4), being an identity in $X$ and $B$, implies that $A$ is a function of $B$. Thus, Eq. (A4) can be written as

$$
\ln A(B)+\ln F(X)=\ln F(B X) .
$$

Differentiating with respect to $B$,

$$
A^{\prime}(B) / A(B)=X F^{\prime}(B X) / F(B X) \text {. }
$$

Changing variable by setting $X=Y / B$,

$$
\left(A^{\prime} B / A\right) / Y=F^{\prime}(Y) / F(Y) \text {. }
$$

Integration with respect to $Y$ yields the desired solution up to a constant factor

$$
F(Y)=Y^{n}
$$

for some constant exponent $n$. Setting $Y=\operatorname{det} D$, we obtain Eqs. (A2) and (A1) as claimed. In summary, from scaling covariance we have narrowed down acceptable metrics to the choices [Eq. (A1)] with unspecified exponent $n$.

\section{APPENDIX B: DIFFUSIVITY COEFFICIENTS IN POLAR COORDINATES}

The seven $\mathcal{D}$ and $\mathcal{E}$ coefficients introduced in Eqs. (38) and (39) are listed here for completeness. Evaluated at $r=R$, they are

$$
\begin{gathered}
\mathcal{D}_{r r}=D_{11}\left(z^{\prime}\right)^{2}-2 D_{13} x^{\prime} z^{\prime}+D_{33}\left(x^{\prime}\right)^{2}, \\
\mathcal{D}_{r \theta}=\frac{2}{R}\left\{D_{11} x^{\prime} z^{\prime}+D_{13}\left[\left(z^{\prime}\right)^{2}-\left(x^{\prime}\right)^{2}\right]-D_{33} x^{\prime} z^{\prime}\right\},
\end{gathered}
$$




$$
\begin{gathered}
\mathcal{D}_{\theta \theta}=\frac{1}{R^{2}}\left[D_{11}\left(x^{\prime}\right)^{2}+2 D_{13} x^{\prime} z^{\prime}+D_{33}\left(z^{\prime}\right)^{2}\right] \\
\mathcal{D}_{r}=R \mathcal{D}_{\theta \theta} \\
\mathcal{D}_{\theta}=-\frac{1}{R} \mathcal{D}_{r \theta}, \\
\mathcal{E}_{r}=-\left(\partial_{i} D_{i 1}\right) z^{\prime}+\left(\partial_{i} D_{i 3}\right) x^{\prime} \\
\mathcal{E}_{\theta}=-\frac{1}{R}\left[\left(\partial_{i} D_{i 1}\right) x^{\prime}+\left(\partial_{i} D_{i 3}\right) z^{\prime}\right]
\end{gathered}
$$

\section{APPENDIX C: PLOTTING THE GEODESIC PATH LENGTH}

In this appendix we take the anchor points at $x_{0}=0\left(G^{\prime}\right.$ $=G$ ) for the sake of illustration. From Eqs. (10), (14), and (21), we find the geodesic path length

$$
\mathcal{L}=2 \int \sqrt{f} d l=2 G \int \sqrt{l^{2}+A^{2}} d l
$$

between $l=[0, A \sinh (L / A)][$ cf. Eq. $(22)$ for $z=(0, L)]$. The result is

$$
\frac{\mathcal{L}}{L}=\frac{A}{L} \sinh (L / A)+\frac{1}{\cosh (L / A)} .
$$

To eliminate $L / A$ in favor of $G L$, we use Eq. (31) with $x_{0}$ $=0$,

$$
\frac{1}{G L}=\frac{A}{L} \cosh \frac{L}{A} .
$$

We then plot $\mathcal{L} / L$ parametrically from these two formulas with $L / A$ as the running parameter; the result is displayed in Fig. 6.

\section{APPENDIX D: NORM OF THE METRIC TENSOR FROM SIMULATIONS}

The metric tensor, as postulated in Eq. (7), can be written somewhat more generally as

$$
g_{i j}=(\operatorname{det} D)^{n}\left(D^{-1}\right)_{i j} .
$$

The exponent $n$ has been specialized to $n=1$ in the postulate. How strong is the evidence for that value? As we have seen, local filament statics requires $n=1$; in addition, this choice produces excellent agreement between theory and simulation. However, it would desirable to have a quantitative error estimate around the value of $n$. This is the purpose of the present appendix, which reexamines the curve and points of Fig. 4. A family of theoretical $s / L$ versus $G^{\prime} L$ curves is displayed in Fig. 9 for several values of $n$; the simulation points of Fig. 4 are shown for comparison. Agreement is seen to require $n=1$ within $2 \%$.

The recalculation of the curve for alternative $n$ values uses Eq. (10), modified to read

$$
f(x)=(1+G x)^{3 n-1} .
$$

In the geodesic calculations [Eqs. (16) and (17)] the right sides then only need to be multiplied by the factor $(3 n$ $-1) / 2$. Taking $x_{0}=0$ for simplicity and following the procedure of Sec. V, we find the following approximate modifications to Eqs. (29) and (31):

$$
\begin{gathered}
\frac{s}{L}=\frac{A}{L}\left[\left(\cosh \frac{L}{A}\right)^{2 /(3 n-1)}-1\right], \\
\frac{1}{G^{\prime} L}=\frac{A}{L}\left(\cosh \frac{L}{A}\right)^{2 /(3 n-1)} .
\end{gathered}
$$

Parametric plotting of these two equations, with $A$ as the running parameter, yields the curves of Fig. 9. From the figure we conclude that $n=1.00 \pm 0.02$.
[1] J. P. Keener, Physica D 31, 269 (1988).

[2] J. Keener and J. Tyson, SIAM Rev. 34, 1 (1992).

[3] R. A. Gray, A. M. Pertsov, and J. Jalife, Nature (London) 392, 75 (1998).

[4] M. Vinson, A. M. Pertsov, and J. Jalife, Physica D 72, 119 (1994).

[5] J. Jalife, O. Berenfeld, A. Skanes, and R. Mandapati, J. Cardiovasc. Electrophysiol. 9, S2 (1998).

[6] M. D. Lesh, J. F. Spear, and E. N. Moore, Cardiac Electrophysiology from Cell to Bedside, 4th ed. (Saunders, Philadelphia), Chap. 40, pp. 364-376.

[7] M. Wellner, A. M. Pertsov, and J. Jalife, Phys. Rev. E 59, 5192 (1999).

[8] M. Wellner, O. Berenfeld, J. Jalife, and A. M. Pertsov, Proc. Natl. Acad. Sci. U.S.A. 99, 8015 (2002).

[9] O. Berenfeld, M. Wellner, J. Jalife, and A. M. Pertsov, Phys. Rev. E 63, 061901 (2001).

[10] O. Berenfeld, M. Wellner, and A. M. Pertsov, Int. J. Bifurca- tion Chaos Appl. Sci. Eng. 13, 3723 (2003).

[11] K. H. W. J. ten Tusscher and A. V. Panfilov, Phys. Rev. Lett. 93, 108106 (2004).

[12] H. Verschelde, H. Dierckx, and O. Bernus, Phys. Rev. Lett. 99, 168104 (2007).

[13] H. Dierckx, O. Bernus, and H. Verschelde, Physica D 238, 941 (2009).

[14] V. N. Biktashev, A. V. Holden, and H. Zhang, Philos. Trans. R. Soc. London, Ser. A 347, 611 (1994).

[15] M. Wellner, O. Berenfeld, and A. M. Pertsov, Phys. Rev. E 61, 1845 (2000).

[16] A. M. Pertsov and M. Vinson, Philos. Trans. R. Soc. London, Ser. A 347, 687 (1994).

[17] D. Barkley, M. Kness, and L. S. Tuckerman, Phys. Rev. A 42, 2489 (1990).

[18] S. Weinberg, Gravitation and Cosmology (Wiley, New York, 1972), pp. 138-140. 